\title{
Mitochondrial changes in endometrial carcinoma: Possible role in tumor diagnosis and prognosis (Review)
}

\author{
ANTONELLA CORMIO ${ }^{1}$, GENNARO CORMIO ${ }^{2,3}$, CLARA MUSICCO ${ }^{4}$, ANNA MARIA SARDANELLI ${ }^{5,6}$, \\ GIUSEPPE GASPARRE $^{7}$ and MARIA NICOLA GADALETA ${ }^{4}$
}

${ }^{1}$ Department of Biosciences, Biotechnologies and Biopharmaceutics, University of Bari, Bari, Italy; ${ }^{2}$ Gynecologic Oncology Unit, IRCCS Istituto Oncologico 'Giovanni Paolo II', Bari, Italy; ${ }^{3}$ Department of Biomedical Sciences and Medical Oncology, University of Bari, Bari, Italy; ${ }^{4}$ CNR-Institute of Biomembranes and Bioenergetics, Bari, Italy; ${ }^{5}$ Department of Basical Medical Sciences, Neurosciences and Sensory Organs, University of Bari, Bari, Italy; ${ }^{6} \mathrm{CNR}$-Institute of Translational Pharmacology, Rome, Italy; ${ }^{7}$ Department of Medical and Surgical Sciences, Unit of Medical Genetics, University of Bologna, Bologna, Italy

Received September 18, 2014; Accepted November 21, 2014

DOI: 10.3892/or.2014.3690

\begin{abstract}
Endometrial carcinoma (EC) is a solid neoplasia for which a role for mitochondria in cancer progression is currently emerging and yet represents a diagnostic and prognostic challenge. EC is one of the most frequently occurring gynecological malignancies in the Western world whose incidence has increased significantly during the last decades. Here, we review the literature data on mitochondrial changes reported in EC, namely, mitochondrial DNA (mtDNA) mutations, increase in mitochondrial biogenesis and discuss whether they may be used as new cancer biomarkers for early detection and prognosis of this cancer.
\end{abstract}

\section{Contents}

1. Introduction

2. Mitochondrial genetics

3. mtDNA mutations in cancer

4. Increase in mitochondrial biogenesis in endometrial hyperplasia and type I EC

5. Association between pathogenic mtDNA mutations altering complex I and increase in mitochondrial biogenesis (oncocytic foci) in type I EC

6. Estrogen stimulation may be responsible for the high frequency of mtDNA mutations and increase in mitochondrial biogenesis in type I EC

Correspondence to: Dr Antonella Cormio, Department of Biosciences, Biotechnologies and Biopharmaceutics, University of Bari, Via Orabona, Bari I-470125, Italy

E-mail: antonella.cormio@uniba.it

Key words: endometrial carcinoma, mitochondrial DNA mutations, mitochondrial biogenesis, prognostic and diagnostic markers
7. mtDNA mutations within the progression model of EC

8. Tumor-specific mtDNA mutations: An additional diagnostic tool to reveal the synchronous nature of simultaneously detected endometrial and ovarian cancer

9. Conclusions

\section{Introduction}

Endometrial carcinoma (EC) is a neoplasia for which a role for mitochondria in cancer progression is currently emerging. EC is the most common malignancy of the female genital tract. This cancer is usually grouped into 2 subsets: endometrioid carcinoma (type I, estrogen-dependent) and non-endometrioid carcinoma (type II, estrogen-independent). Type I is the most common form, displaying a less aggressive behavior than type II. The development of type I endometrial cancer is considered a multistep process, with slow progression from normal endometrium to hyperplasia and endometrial cancer as a result of an unopposed estrogenic stimulation $(1,2)$.

Mitochondria are essential organelles in all eukaryotic cell systems, and are the powerhouses to provide ATP for a multitude of cellular processes by the oxidative phosphorylation (OXPHOS) system. They are the hub of metabolic pathways, primary sources of reactive oxygen species (ROS), regulators of apoptosis as well as signal transduction regulators and buffers of intracellular calcium (3-5). In addition, ATP production has to be continually adapted to cover current requirements, with marked changes in production levels in tissues alternating between different states (6).

Many years ago Otto Warburg observed that cancer cells actively metabolize glucose and produce an excess of lactate even in the presence of oxygen, the so-called reverse Pasteur effect or aerobic glycolysis. He hypothesized that malignant cells should harbor defects in the respiratory chain of mitochondria and uncovered that, although ATP production via glycolysis is a less efficient process, the increased glycolytic rate in cancer cells largely compensates the lower energy yield per single glucose molecule (7). Recently, it was observed 
that different cancer cell types undergo different bioenergetic alterations and that a constant metabolic remodeling takes place according to the stages of cancer progression; thereby, tumors may be glycolytic, partial mitochondrial OXPHOSdependent or complete OXPHOS-dependent $(8,9)$.

Numerous studies have reported changes in the mitochondrial number (10), in mitochondrial DNA (mtDNA) content (11), in respiratory chain activity and in mitochondrial gene expression $(9,12)$ in different human cancers. Different somatic mtDNA mutations have been reported in various types of human tumors $(13,14)$. In addition to mutations that directly affect mtDNA, mutations in nuclear DNA affecting mitochondrial proteins such as tricarboxylic acid cycle genes (succinate dehydrogenase, fumarate hydratase and isocitrate dehydrogenase1 and 2) have been described (15-18).

This review aims to present the literature data on mitochondrial alterations in EC discussing the possible role of these alterations in EC diagnosis and prognosis. The reported data refer mostly to type I EC which accounts for the majority of cases and has been therefore more extensively investigated.

\section{Mitochondrial genetics}

Human mitochondria contain a small DNA, mtDNA, of $\sim 16,569$ bp (19) that codes for 2 rRNAs (12 and 16S), 22 tRNAs and 13 protein subunits of 4 of the 5 complexes of the respiratory chain. These proteins include: 7 subunits of NADH dehydrogenase (complex I), one of cytochrome $c$ reductase (complex III), 3 of cytochrome $c$ oxidase (complex IV) and 2 of ATP synthase (complex V). The non-coding region, the so-called D-loop region, is $\sim 1.1 \mathrm{kbp}$ long. It is the most variable part of the genome and contains regulatory signals for replication and transcription $(20,21)$. The majority of mitochondrial proteins $(\sim 1500)$ are coded by nuclear DNA, transcribed and translated in the cytoplasm and then transported into the mitochondria. The mitochondrial genetic system has peculiar features with respect to the nuclear one. mtDNA is maternally inherited and is present in a large number of copies per cell $(\sim 2-4000$, polyploidy) $(22,23)$. It is more susceptible to ROS-induced mutations (point mutations or deletions) than nuclear DNA since it is located close to the mitochondrial respiratory chain, the major source of ROS in the cell (24). mtDNA point mutations may be simple neutral polymorphisms with no important functional consequences for the cell or deleterious mutations potentially dangerous for the cell. The mtDNA in a cell or in a tissue of a single individual may be all of the same type, wild-type or mutant (homoplasmy) or different genotypes may coexist (heteroplasmy). Therefore, it is important to know what percentage of mutant mtDNA molecules (threshold) can lead to a dysfunction of the mitochondrial respiratory apparatus $(25,26)$.

The sequential accumulation of de novo mtDNA point mutations in an individual mtDNA molecule generates a mtDNA haplotype. A group of related haplotypes, acquired along radiating maternal lineages, gives rise to haplogroups which tend to be restricted to specific geographic areas and/or ethnic groups (27). These ancient mtDNA variants related to haplogroups may influence individual predisposition to diseases (28).

\section{3. mtDNA mutations in cancer}

mtDNA point mutations and deletions have been extensively described in solid tumors. However, the functional relevance of these mtDNA changes in tumor formation and/or promotion is a matter of debate. Point mtDNA mutations could arise either in the female germ line (germline mutations) and predispose to cancer or in the mtDNA molecules of the affected tissues (tumor-specific somatic mutations) and participate in the tumor progression process. As suggested by Brandon et al (29) tumor-specific somatic mutations may be classified as tumorigenic and adaptive. The tumorigenic mutants are severe mutations (i.e. disruptive mutation, nonsense or frame-shift mutations) that alter the respiratory chain and may increase mitochondrial ROS production. ROS promote neoplastic transformation since they diffuse in the nucleus and induce mutations in genes which regulate cell replication, in proto-oncogenes and in tumor suppressor genes. Adaptive mtDNA mutations are instead mild mutations that allow an adaptation of cancer to adverse environments by participating in the metabolic remodeling recently included among cancer hallmarks (30). In fact, mtDNA-encoded proteins are de facto metabolic enzymes, whose function impinges directly on the same pathways as the well-known tumor suppressors succinate dehydrogenase, fumarate hydratase and isocitrate dehydrogenase (whose suppressor vs. oncogenic role is still debated) of the Krebs cycle. These genes have long been shown to contribute to tumorigenesis via stabilization of hypoxia inducible factor $1 \alpha(\mathrm{HIF} 1 \alpha)(31)$, thereby likely driving the glycolytic shift that tightly depends on this transcription factor. Similarly, adaptive mtDNA mutations may influence tumor progression conferring to cancer the ability to metastasize.

Germline mtDNA mutations in EC: Predisposing or protective modifiers of tumorigenesis? Screening tools that may enable to select populations at a high risk for EC and support the process of prevention and early diagnosis have been developed in recent years. The most common risk factors associated with the development of EC are: exposure to unopposed endogenous estrogen, as occurs in chronic anovulation (polycystic ovary syndrome), tamoxifen treatment, obesity, hypertension, type II diabetes, age, nulliparity, infertility, early age of menarche and late age of menopause (32). A familial risk for developing EC was found in women with Lynch syndrome or hereditary nonpolyposis colon cancer (HNPCC). This autosomal dominant syndrome is characterized by a germline mutation in one of the mismatch repair genes: MLH1, MSH2 and MSH6 (33). The association between germline mutations in the BRCA genes and the risk of EC remains controversial (34). Since mtDNA mutations have been described in EC, they have also been correlated with cancer risk. In particular, Liu et al reported that the m.16189T $>C$ base change in the D-loop region was associated with susceptibility to EC (35). A study on a southwest China population pointed out that the mitochondrial polymorphisms associated to haplogroup D (such as m.5178A $>C$ in the $M T-N D 1$ gene) predispose to EC (36). Recently, in the Polish population, 3 polymorphisms of mtDNA, namely, $\mathrm{m} .16223 \mathrm{C}>\mathrm{A}, \mathrm{m} .207 \mathrm{G}>\mathrm{A}$ and $\mathrm{m} .16126 \mathrm{~T}>\mathrm{C}$ in the D-loop region, were associated with an increased risk of EC, while 
haplogroup $\mathrm{H}$, with its defining polymorphism $\mathrm{m} .7028 \mathrm{C}>\mathrm{T}$ in the COI gene, appeared to be a cancer protective factor (37). However, most of such studies have reported mere associations and lack functional proof that certain mitochondrial variants, many of which are common polymorphisms and even haplogroup-defining, may truly impinge on EC predisposition or be protective modifiers of EC tumorigenesis. Overall, it is interesting that evolution may have selected and fixed within certain populations advantageous nucleotide changes, perhaps able to allow a more efficient metabolism, that, however, as a double-edged sword, may facilitate EC occurrence. Functional studies with cybrid cells, i.e. cells in which different mtDNA genotypes may be investigated independently from the nuclear background, will help to clarify what evolutionary advantages these variants may present. However, if the variants reported to increase risk to EC truly constitute a predisposing background, it should be explained why this would occur only in the endometrium, since they are for the largest part homoplasmic within the germline. Moreover, since these polymorphisms are matrilinearly inherited, it would be of utmost importance to monitor the daughters of patients to verify whether such risk is increased in the generations to which the haplotype is passed on.

Somatic mtDNA mutations in EC: Possible molecular markers for cancer detection, diagnosis and prognosis. In EC, the prognosis still relies on conventional pathological features such as histological type and grade, as well as myometrial or lymphovascular space invasion. Unfortunately this analysis still does not provide a relevant prognosis. Many molecular biomarkers have also been defined (i.e. PTEN, K-ras, PT53, catenin, MSH2 and MSH6). However, not a single universally accepted predictive or prognostic marker exists to date to assist clinical decisions regarding EC (38).

Studies on somatic mtDNA mutations to ascertain their role as possible molecular markers for cancer detection, diagnosis and prognosis of EC have also been performed. Initial studies analyzed scattered regions of mtDNA, particularly the regulatory D-loop region and the 2 rRNA genes. Changes in length of short base-repetitive sequences of mtDNA (mitochondrial microsatellite instability, mtMSI), particularly in the D-loop region (at m.310, 564 and 16184) and in the $12 \mathrm{~S}$ rRNA gene (m.956) were found in $50 \%$ of the EC analyzed samples $(39,40)$. The mtMSI frequency in EC was found to be significantly higher (48.4\%) than this frequency in cervical (25.4\%), ovarian (21.9\%) and breast cancer (29.4\%) (40). However, Wang et al (41), while analyzing the occurrence of somatic mtDNA mutations in EC cells harvested by laser-capture microdissection from separated areas of the same tumor and from adjacent normal cells, demonstrated that different mtMSI genotypes were observed in different histological areas. This suggested, according to the authors, that these mtDNA alterations occurred randomly and independently in individual cells or in a small group of cells and that they may be a secondary event in progression, rather than, likely, predisposing genetic lesions. Somatic mtDNA mutations in the mtDNA D-loop region were detected also in $63 \%$ of type II EC analyzed patients (42).
The common deletion of human mtDNA, a 4977 bp deletion, was also analyzed in EC tissue. The mean mutated/wild-type mtDNA ratio was found significantly higher in the control group compared to the cancer group so that the accumulation of the deletion seemed to be associated with the aging process rather than with cancer (43).

Semczuk et al (44) studied the relationship between somatic mtDNA mutations and clinical and pathological variables of women affected by EC. The sequencing of small regions of mtDNA (nucleotides 135-433, 2986-3301, 4981-5500, 10390-10700 and 12005-12386), including part of the D-loop region, $16 \mathrm{~S}$ rRNA, the $M T-N D 4 L$ gene and different tRNAs was performed in hyperplastic and cancer tissues. Somatic mtDNA mutations were not detected in hyperplastic endometrial tissues, however, they were found in $10 \%$ of analyzed patients and were unrelated to their clinicopathological data (age, clinical stage, histological grade and type or depth of myometrial invasion) (44).

The relationship between mtMSI at m.310, 564, 16184 and mtDNA content was also investigated in EC cells to verify whether the increased instability of the D-loop region may induce an alteration in mtDNA copy number since this mtDNA region contains regulatory signals for replication and transcription. In EC cells a correlation between the increase in the mtDNA copy number and the presence of mtMSI was found significantly high only at m.303 (45). However, caution is warranted, in assigning a role for homopolymeric stretch instability, such as that which occurs at positions m.309-315, in the mtDNA copy number increase, as it is also plausible to assume that the instability may be a consequence of an excessive replication due, for instance, to estrogen stimulation or other mitochondrial biogenesis stimuli.

In a recent study Guerra et al (46) sequenced the entire mtDNA in order to have a more informative picture of somatic mtDNA mutations present in EC tumor specimens, including mutations in the coding regions of mtDNA. In type I EC samples and in matched typical hyperplastic samples as control all tumor-specific (exclusively present in tumor mtDNA), non-tumor-specific (present in both the tumor and the hyperplasia mtDNA) and hyperplasia-specific (exclusively present in hyperplasia mtDNA) variants were detected and annotated. Tumor-specific mtDNA mutations were found in $69 \%$ of the analyzed cancer endometrial samples. Many of these mutations (59\%) were predicted to be pathogenic by in silico analysis and had not been reported in the literature. Interestingly, these types of mutations were absent in hyperplastic tissues: all mtDNA variants detected in hyperplasia were in fact clear haplogroup determinants. A correlation between occurrences of mtDNA mutations and grading was not found, although, a clear-cut tendency for low-grade (G1-G2) compared with high-grade (G3) tumors to harbor clearly pathogenic mtDNA was observed. No other correlation between the occurrence of mtDNA mutations and other clinical data was found.

The sequencing of the entire mtDNA molecules in EC tumors and matched hyperplastic tissues allowed detection in a higher number of patients $(70 \%)$ of a higher number of mtDNA mutations than previously reported. This frequency is very high compared to the other EC molecular biomarkers (38). 
Furthermore, the presence of mtDNA mutations only in cancer tissues allowed distinguishing tumor vs. hyperplastic and non-malignant tissues. Therefore, mtDNA mutations can be considered useful biomarkers for cancer detection.

\section{Increase in mitochondrial biogenesis in endometrial hyperplasia and type I EC}

Mitochondrial biogenesis usually refers to an increase in the mitochondrial number and/or of the mitochondrial mass per cell. The cell increases mitochondrial biogenesis to overcome a bioenergetics deficit or to face an increased energy requirement (reviewed in refs. 47-49).

The number of mitochondria is generally expressed by the cellular content of mtDNA measured as mtDNA/nuclear DNA ratio whereas citrate synthase (CS) activity is considered a reliable marker of mitochondrial mass (50). The master regulator of mitochondrial biogenesis is the nuclear transcriptional coactivator PPAR $\gamma$-coactivator-1 $\alpha$ (PGC-1 $\alpha$ ) (51). This factor co-activates the nuclear respiratory factors 1 and 2 (NRF-1 and NRF-2) that, in turn, stimulate the expression of a large number of nuclear genes involved in mitochondrial respiration and in mtDNA replication and transcription (52). One of these genes, the one coding for mitochondrial transcription factor A (TFAM), regulates both mtDNA transcription and mtDNA replication (53).

In different human cancers, changes in the number of mitochondria (10) and/or in the level of mtDNA (54) have been reported. A 2 -fold increase in mtDNA copy number was found in EC compared to normal endometrial glandular epithelial cells collected by laser capture microdissection (45). Moreover, a 2-fold increase in mtDNA content and in CS activity as well as TFAM, NRF-1 and PGC-1 $\alpha$ protein content was found by our group (55) in a pooled group of type I EC endometrial tissues compared to a pooled group of endometrial proliferative control tissue. These results suggested an increase in mitochondrial biogenesis associated with the upregulation of the PGC- $1 \alpha$ signaling pathway in type I EC tissue. The increase of mitochondrial biogenesis was evaluated also in hyperplastic endometrium since endometrial hyperplasia often precedes type I EC. The endometrial hyperplasia can be subdivided into hyperplasia with cytological atypia (atypical hyperplasia) and hyperplasia lacking these features (typical hyperplasia). The former frequently progresses to a well-differentiated type I EC (56). The mtDNA content and CS activity were measured in benign endometrial, in hyperplastic and in cancer tissue. It was found that the mtDNA content and CS activity increased in hyperplastic endometrium compared to the control tissues, even when their level remained lower compared to cancer tissue. mtDNA content increase preceded an increase in CS activity. In fact, mtDNA began to increase significantly already in typical hyperplasia while CS activity increased significantly only in atypical hyperplasia (57).

The increases in mtDNA content and CS activity could be envisioned in type I EC as potential molecular markers to establish the risk of malignant transformation of endometrial hyperplasia and may have a clinical value in patient management. However, due to the interindividual variability, a comparison of each hyperplastic tissue to matched control tissue is required. Further analysis in a higher panel of patients and prospective longitudinal studies are necessary to address this topic.

\section{Association between pathogenic mtDNA mutations altering complex I and increase in mitochondrial biogenesis (oncocytic-like foci) in type I EC}

It has been reported that a specific subset of tumors, namely oncocytic neoplasias, is characterized by the pathological hallmark of aberrant mitochondrial hyperproliferation. Oncocytic tumors harbor high loads of disruptive (nonsense or frameshift) mtDNA mutations, the large majority of which map in respiratory complex I genes and are associated with a disassembly of complex I (58-60). The mitochondrial hyperproliferation in these tumors has been hypothesized to be a compensatory effect triggered in response to a retrograde signaling from dysfunctional mitochondria to the nucleus (reviewed in refs. 61-63).

The association between mtDNA mutations in respiratory complex I genes, inefficiency of complex I and mitochondrial biogenesis was also verified in type I EC biopsies (46). In fact, in $72 \%$ of EC samples, previously characterized for tumor-specific mtDNA mutations by the sequencing of the entire mitochondrial genome, oncocytic-like foci were found. An immunohistochemical (IHC) staining of respiratory complex subunits revealed in $69 \%$ of EC samples with oncocytic-like foci, a partial or total loss of staining for the NDUFB8 subunit of complex I suggesting at least a partial disassembly of complex I and confirming an association between the loss of the complex and oncocytic-like transformation. Approximately $70 \%$ of EC patients with loss of complex I harbored pathogenic mtDNA mutations, the majority in respiratory complex I genes. Moreover, in EC samples compared to matched hyperplastic tissues, an increase in the content of mtDNA, in structural mitochondrial proteins TFAM and porine, in some nuclear DNA encoded respiratory subunits NDFUA9, SDHA, SDHB, Core II and mitochondrial antioxidant enzymes (Prx3 and MnSOD) was found, especially, in the EC samples harboring pathogenic tumorspecific mtDNA mutations. Oncocytic-like foci found in $72 \%$ of analyzed patients confirmed the increase of mitochondrial biogenesis already found by analyzing a pooled group of EC samples (46). The increase in mitochondrial biogenesis and antioxidant enzymes may be an attempt of EC tissues to overcame bioenergetic deficit and ROS production due to alteration of complex I, the major source of cellular ROS (64-66).

Previous studies indicate that most oncocytic tumors, harboring disruptive mtDNA mutations, retain a low-proliferating, benign behavior because of their inability to undergo adaptation to hypoxia and because of their deranged respiratory metabolism $(59,67,68)$. Since type I EC prognosis is generally more favorable than that of type II EC, it can be hypothesized that in type I EC a combined action of deranged respiratory metabolism due to mtDNA mutations and an unopposed estrogen stimulation may induce mitochondrial proliferation, as found in oncocytic-like foci, thus contributing to maintain the tumor in a less aggressive stage. Studies on mitochondrial biogenesis in estrogen-independent type II EC are warranted to confirm whether this hypothesis holds true. 


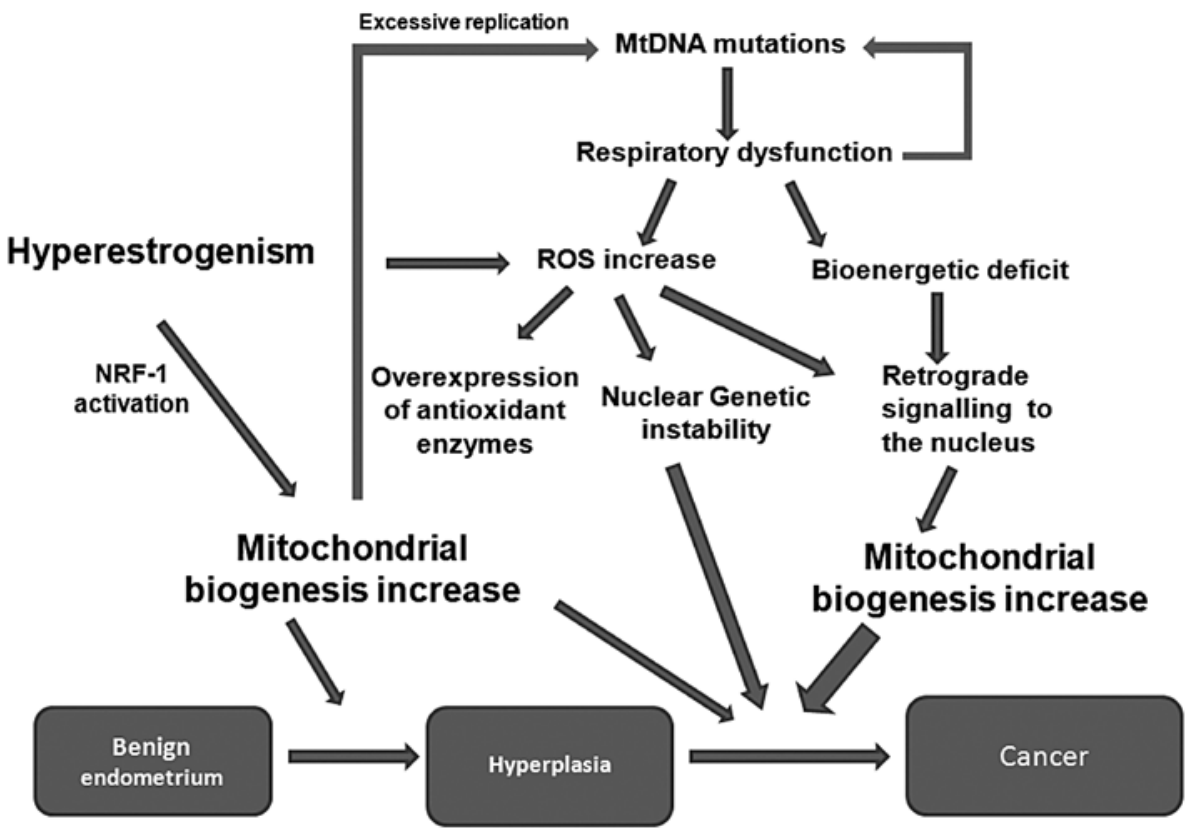

Figure 1. Effect of hyperestrogenism in the progression from benign endometrium to hyperplasia to cancer. A mild increase in mitochondrial biogenesis occurs endometrial hyperplasia and cancer because of a direct interaction of estrogens with NRF-1. In cancer tissue the effect of estrogen stimulation on the increase in mitochondrial biogenesis is reinforced by the occurrence of pathogenic mtDNA mutations. These mutations, generated by estrogen-related ROS increase and by excessive mtDNA replication, may reach a threshold value and affect respiratory complexes, in particular complex I. The consequent bioenergetic deficit and ROS increase may trigger a retrograde signaling to the nucleus that, through the upregulation of the PGC-1 $\alpha$ signaling pathway, stimulates mitochondrial proliferation and overexpression of antioxidant enzymes as a compensatory response. ROS may also induce mutations in mtDNA and in proto-oncogenes and tumor suppressor genes leading to nuclear genetic instability and to cancer. NRF-1, nuclear respiratory factor 1; mtDNA, mitochondrial DNA; ROS, reactive oxygen species.

\section{Estrogen stimulation may be responsible for the high frequency of mtDNA mutations and increase in mitochon- drial biogenesis in type I EC}

The role of estrogens in mitochondrial function is well established suggesting an important role in maintaining mitochondrial structure and function (69). The genomic activity of estrogens is mediated by $\operatorname{ER} \alpha$ and $\operatorname{ER} \beta$ that are members of the steroid/nuclear receptor superfamily of transcription factors. $E R \alpha$ and $E R \beta$ have been identified in mitochondria and bind to the D-loop of mouse and human mtDNA (70). However, mechanisms by which ERs coordinate the complex signal pathways between the membrane, mitochondria and nucleus remain to be fully determined.

Estrogen can also inhibit mitochondrial apoptosis since it increases anti-apoptotic Bcl-2 and can affect mitochondrial dynamics altering the fusion/fission ratio. Moreover, it was reported that estradiol stimulates mitochondrial biogenesis, in particular, increases mtDNA content in breast and lung adenocarcinoma cells by stimulating directly NRF-1 gene expression and consequently increasing TFAM, mitochondrial transcription and oxygen consumption (71). In human breast cancer cells, on the other hand, estradiol produces high rates of mitochondrial ROS that act as signal transducing factors activating NRF-1 (72).

It can be envisioned that estrogen may favor the appearance of mtDNA mutations by 2 mechanisms: 1) stimulating mitochondrial biogenesis: excessive mtDNA replication, may favor replication errors and consequently mtDNA mutations; 2) inducing mitochondrial ROS: ROS may directly damage
mtDNA and generate mtDNA instability. In cancer tissue, but not in hyperplasia, these mtDNA mutations, may reach detectable value probably due to positive selection and/or to tumor clonality. These mutations, above a threshold, may lead to respiratory dysfunction, in particular of complex I and consequently a bioenergetic deficit and ROS increase that generate, in turn, further mtDNA mutations.

In Fig. 1 a possible explanation of the effects of estrogen stimulation in progression from benign endometrium to hyperplasia and to cancer is schematically reported.

In hyperplastic tissue, which undergoes estrogen stimulation (56) and where potentially pathogenic mtDNA mutations have not been found, a mild increase in mitochondrial biogenesis takes place due to estrogen stimulation. In type I EC cancer tissues, a synergistic mechanism may be envisioned in which the effect of estrogen stimulation on the increase in mitochondrial biogenesis may be reinforced by the occurrence of pathogenic mtDNA mutations. In cancer these mutations, above a threshold, may lead to respiratory dysfunction and consequently to a bioenergetic deficit and ROS increase. They may trigger a retrograde signaling to the nucleus that, through the upregulation of the PGC- $1 \alpha$ signaling pathway, may stimulate mitochondrial biogenesis and induce an increase in antioxidant enzymes as a compensatory response (47). ROS may induce mutations in mtDNA and in proto-oncogenes and tumor suppressor genes leading to nuclear genetic instability. It can be envisioned, therefore, that in type I EC, hyperestrogenism, through an increase in mtDNA mutations and mitochondrial biogenesis, may have a role in neoplastic transformation. 


\section{7. mtDNA mutations within the progression model of EC}

The screening of point mutations in 4 oncogenes commonly involved in type I EC pathogenesis, namely PTEN, KRAS, CTNNB1 and TP53 in the same patients in which mtDNA mutations were analyzed, allowed to place, for the first time, mtDNA mutations within the Vogelstein-like progression model of EC (46). In particular, it was observed that both canonical nuclear hits and mtDNA mutations occurred after hyperplastic development and before progression to high grade EC. In line with this, pathogenic mtDNA mutations were observed preferentially in low-grade (G1-G2) compared with high-grade (G3) tumors. Furthermore, the percentage of the analyzed patients harboring mtDNA mutations was higher compared to that of patients with mutations in oncogenes/tumor suppressors suggesting that mtDNA mutations may precede the genetic instability of nuclear genes (46). If so, an increase in ROS may be responsible for nuclear DNA damage and may induce genetic instability (Fig. 1) as reported in thyroid tumors (73). These results may be suggestive of a role of mtDNA mutations in the transition from simple hyperplasia to neoplasia. However, it should be considered that even if a high percentage (70\%) of EC patients harbor tumorspecific mtDNA mutations, several tumor-specific mtDNA mutations were not potentially pathogenic and finally that not all mutations were homoplasmic or presented a mutation load over $50 \%$, a threshold likely implying a phenotypic effect. Therefore, we cannot exclude that they may be regarded as side-effects of tumorigenesis, which may probably not impinge on tumor progression. More research is needed to assign a role to mtDNA mutations in tumor progression.

\section{Tumor-specific mtDNA mutations: An additional diagnostic tool to reveal the synchronous nature of simultaneously detected endometrial and ovarian cancer}

Recently, it was reported that the sequencing of the entire mtDNA helps to discriminate between the independent and metastatic origin of simultaneously detected tumors of the endometrium and ovary when histopathological criteria and canonical molecular methods fail or are ambiguous. The sequencing of the entire mtDNA from endometrial and ovarian cancer tissues of the same patient allows a comparison of tumor-specific mtDNA mutations eventually present in both tumors. The presence of one or more tumor-specific mtDNA mutations common to both tissues suggests a common clonal origin of the somatic mutation and the metastatic origin of 1 of the 2 cancers. On the contrary, the absence of tumorspecific somatic mtDNA mutations common to both tissues indicates an independent synchronous origin of the 2 cancers. In fact, it is very unlikely that the same somatic mutation may arise synchronously and independently in 2 tumors. This approach was applied for the first time to a patient carrying two simultaneously detected tumors in ovary and endometrium and a pelvic lymph node metastasis. Sequencing of the entire mtDNA molecule from the 3 different tissues of the patient revealed a frameshift deletion in the ND4 gene exclusively in the ovarian cancer, suggesting a different clonal origin of EC and lymph node metastasis. A comparative genome hybridization analysis revealed a duplication of $1 \mathrm{qXq}$ only in EC and lymph node metastasis suggesting that the lymph node metastasis was derived from the EC (74). Very recently the same approach was implemented in 11 additional cases of simultaneously detected endometrial and ovarian cancer. Histopathological criteria and canonical molecular analysis such as microsatellite instability, B-catenin immunohistochemical staining and CTNNB1 mutation screening provided ambiguous diagnoses in certain cases. The mtDNA genotype approach allowed to define the diagnosis in half of the analyzed cases (75). This new molecular approach has the advantages of requiring small amounts of starting material and has relatively low costs. However, it should be taken into account that not all analyzed tumor samples may harbor tumor-specific mtDNA mutations, although given the high percentage of EC samples with an accumulation of variants, this molecular method appears to be particularly suitable to aid diagnoses.

\section{Conclusion}

mtDNA mutations and mitochondrial proliferation are hallmarks of EC. The study of mitochondrial changes in EC, particularly in type I, reveal the following: i) multiple germline mutations are associated with EC, although, currently lack a functional proof that may truly impinge on EC predisposition or are protective modifiers of EC tumorigenesis; ii) somatic tumor-specific mtDNA mutations can be useful biomarkers for the distinction of tumor vs. hyperplastic tissues since they are present in high percentage only in EC; iii) somatic pathogenic mtDNA mutations may participate in the tumor progression process by contributing to genetic instability: they may not have a causative role but be responsible and provide an explanation for the respiratory dysfunction and the presence of oncocytic-like foci; iv) mtDNA mutations can be used to investigate tumor clonality and to discriminate between independent and metastatic tumors; v) the increase in mitochondrial biogenesis in endometrial hyperplasia could be envisioned as a potential biomarker to establish the risk of malignant transformation; vi) the presence of oncocytic-like foci in EC may be a prognostic marker of low-proliferating, more indolent cancer behavior.

In conclusion, studies of mitochondrial changes in EC should be implemented in the future since it requires small amounts of starting material and has a relatively low cost. This may open new horizons in the diagnosis and in the prognosis of EC especially when the screening of other diagnostic markers have failed or provide ambiguous diagnosis. Finally, similar studies in type II EC should be welcome to differentiate the two EC types and to better define the role of hyperestrogenism in this tumor.

\section{Acknowledgements}

This study was supported by the University of Bari grant Fondi Ateneo 2012, to A.C. This study was also supported by the Associazione Italiana per la Ricerca sul Cancro (AIRC) IG14242, by the Fondazione Umberto Veronesi grant DISCO TRIP and in part by the EU ITN-People project MEET (Mitochondrial European Educational Training), to G.G. and by MIUR-PON01_02093, to A.M.S. 


\section{References}

1. Hecht JL and Mutter GL: Molecular and pathologic aspects of endometrial carcinogenesis. J Clin Oncol 24: 4783-4791, 2006.

2. Matias-Guiu X, Catasus L, Bussaglia E, et al: Molecular pathology of endometrial hyperplasia and carcinoma. Hum Pathol 32: 569-577, 2001.

3. Murphy MP: How mitochondria produce reactive oxygen species. Biochem J 417: 1-13, 2009.

4. Brookes PS, Yoon Y, Robotham JL, Anders MW and Sheu SS: Calcium, ATP and ROS: a mitochondrial love-hate triangle. Am J Physiol Cell Physiol 287: C817-C833, 2004

5. Cadenas $\mathrm{E}$ and Davies K: Mitochondrial free radical generation, oxidative stress and aging. Free Radic Biol Med 29: 222-230, 2000.

6. Hüttemann M, Lee I, Samavati L, Yu H and Doan JW: Regulation of mitochondrial oxidative phosphorylation through cell signaling. Biochim Biophys Acta 1773: 1701-1720, 2007.

7. Warburg O: On respiratory impairment in cancer cells Science 124: 269-270, 1956.

8. Bonuccelli G, Tsirigos A, Whitaker-Menezes D, et al: Ketones and lactate 'fuel' tumor growth and metastasis: Evidence that epithelial cancer cells use oxidative mitochondrial metabolism. Cell Cycle 9: 3506-3514, 2010.

9. Smolkova K, Plecita-Hlavata L, Bellance N, Benard G, Rossignol $\mathrm{R}$ and Jezek P: Waves of gene regulation suppress and then restore oxidative phosphorylation in cancer cells. Int J Biochem Cell Biol 43: 950-968, 2011

10. Pedersen PL: Tumor mitochondria and the bioenergetics of cancer cells. Prog Exp Tumor Res 22: 190-274, 1978.

11. Yu M: Generation, function and diagnostic value of mitochondrial DNA copy number alterations in human cancer. Life Sci 89: 65-71, 2011.

12. Baracca A, Chiaradonna F, Sgarbi G, Solaini G, Alberghina L and Lenaz G: Mitochondrial complex I decrease is responsible for bioenergetic dydfunction in K-ras transformed cells. Biochim Biophys Acta 1797: 314-323, 2010.

13. Larman TC, DePalma SR, Hadjipanayis AG, et al: Spectrum of somatic mitochondrial mutations in five cancers. Proc Nat Acad Sci USA 109: 14087-14091, 2012.

14. Kulawiec M, Salk JJ, Ericson NG, Wanagat J and Bielas JH: Generation, function and prognostic utility of somatic mitochondrial DNA mutations in cancer. Environ Mol Mutagen 51: 427-439, 2010

15. Ward PS, Patel J, Wise DR, et al: The common feature of leukemia-associated IDH1 and IDH2 mutations is a neomorphic enzyme activity converting alpha-ketoglutarate to 2-hydroxyglutarate. Cancer Cell 17: 225-234, 2010.

16. Chandra D and Singh KK: Genetic insights into OXPHOS defect and its role in cancer. Biochim Biophys Acta 1807: 620-625, 2011.

17. Picaud S, Kavanagh KL, Yue WW, et al: Structural basis of fumarate hydratase deficiency. J Inherit Metab Dis 34: 671-676, 2011.

18. Bardella C, Pollard P and Tomlinson I: SDH mutations in cancer Biochim Biophys Acta 1807: 1432-1443, 2011.

19. Anderson S, Bankier AT, Barrell BG, et al: Sequence and organization of the human mitochondrial genome. Nature 290 457-465, 1981

20. Attardi G: Animal mitochondrial DNA: an extreme example of genetic economy. Int Rev Cytol 93: 93-145, 1985.

21. Chen X and Butow RA: The organization and inheritance of the mitochondrial genome Nat Rev Genet 6: 815-825, 2005.

22. Lightowlers RN, Chinnery PF, Turnbull DM and Howell N: Mammalian mitochondrial genetics: heredity, heteroplasmy and disease. Trends Genet 13: 450-455, 1997.

23. Johns DR: Paternal transmission of mitochondrial DNA is (fortunately) rare. Ann Neurol 54: 422-424, 2003.

24. DiMauro S, Tanji K, Bonilla E, Pallotti F and Schon EA: Mitochondrial abnormalities in muscle and other aging cells: classification, causes and effects. Muscle Nerve 26: 597-607, 2002.

25. Rossignol R, Faustin B, Rocher C, Malgat M, Mazat JP and Letellier T: Mitochondrial threshold effects. Biochem J 370: 751-762, 2003

26. Musicco C, Cormio A, Calvaruso MA, et al: Analysis of the mitochondrial proteome of cybrid cells harbouring a truncative mitochondrial DNA mutation in respiratory complex I. Mol Biosyst 10:1313-1319, 2014.
27. Torroni A, Achilli A, Macaulay V, Richards M and Bandelt HJ: Harvesting the fruit of the human mtDNA tree. Trends Genet 22: 339-345, 2006

28. Wallace DC: Bioenergetics in human evolution and disease: implications for the origins of biological complexity and the missing genetic variation of common diseases. Philos Trans $\mathrm{R}$ Soc Lond B Biol Sci 368: 2012-2067, 2013.

29. Brandon M, Baldi P and Wallace DC: Mitochondrial mutations in cancer. Oncogene 25: 4647-4662, 2006.

30. Hanahan D and Weinberg RA: Hallmarks of cancer: the next generation. Cell 144: 646-674, 2011.

31. McKenzie M, Lazarou M, Thorburn DR and Ryan MT: Mitochondrial respiratory chain supercomplexes are destabilized in Barth syndrome patients. J Mol Biol 361: 462-469, 2006.

32. Burke WM, Orr J, Mario L, et al: Endometrial Cancer: A review and current management strategies: part I. SGO Clinical Practice Endometrial Cancer Working Group. Gynecol Oncol 134 385-392, 2014.

33. Bonadona V, Bonaïti $\mathrm{B}$, Olschwang $\mathrm{S}$, et al: Cancer risks associated with germline mutations in MLH1, MSH2 and MSH6 genes in Lynch syndrome. JAMA 305: 2304-2310, 2011.

34. Levine DA, Lin O, Barakat RR, et al: Risk of endometrial carcinoma associated with BRCA mutation. Gynecol Oncol 80: 395-398, 2001

35. Liu VW, Wang Y, Yang HJ, et al: Mitochondrial DNA variant $16189 \mathrm{~T}>\mathrm{C}$ is associated with susceptibility to endometrial cancer. Hum Mutat 22: 173-174, 2003.

36. Xu L, Hu Y, Chen B, Tang W, Han X, Yu H and Xiao C: Mitochondrial polymorphisms as risk factors for endometrial cancer in southwest China. Int J Gynecol Cancer 16: 1661-1667, 2006.

37. Czarnecka AM, Klemba A, Semczuk A, et al: Common mitochondrial polymorphisms as risk factor for endometrial cancer. Int Arch Med 2: 33, 2009

38. Matias-Guiu X and Davidson B: Prognostic biomarkers in endometrial and ovarian carcinoma. Virchows Arch 464: 315-331, 2014.

39. Liu VW, Yang HJ, Wang Y, et al: High frequency of mitochondrial genome instability in human endometrial carcinomas. Br J Cancer 89: 697-701, 2003.

40. Wang Y, Liu VW, Tsang PC, et al: Microsatellite instability in mitochondrial genome of common female cancers. Int J Gynecol Cancer 16: 259-266, 2006

41. Wang Y, Xue WC, Liu VW and Ngan HY: Detection of mosaic pattern of mitochondrial DNA alterations in different populations of cells from the same endometrial tumor. Mitochondrion 7: 171-175, 2007.

42. Pejovic T, Ladner D and Intengan M: Somatic D-loop mitochondrial DNA mutations are frequent in uterine serous carcinoma. Eur J Cancer 40: 2519-2524, 2004.

43. Futyma K, Putowski L, Cybulski M, Miotla P, Rechberger T and Semczuk A: The prevalence of mtDNA4977 deletion in primary human endometrial carcinomas and matched control samples. Oncol Rep 20: 683-688, 2008.

44. Semczuk A, Lorenc A, Putowski L, Futyma K, Bryk J, Miotla P and Bartnik E: Clinicoprognostical features of endometrial cancer patients with somatic mtDNA mutations. Oncol Rep 16: 1041-1045, 2006

45. Wang Y, Liu VW, Xue WC, Tsang PC, Cheung AN and Ngan HY: The increase of mitochondrial DNA content in endometrial adenocarcinoma cells: a quantitative study using laser-captured microdissected tissues. Gynecol Oncol 98: 104-110, 2005.

46. Guerra F, Kurelac I, Cormio A, et al: Placing mitochondrial DNA mutations within the progression model of type I endometrial carcinoma. Hum Mol Genet 20: 2394-2405, 2011.

47. Wenz T: Regulation of mitochondrial biogenesis and PGC-1 $\alpha$ under cellular stress. Mitochondrion 13: 134-142, 2013.

48. Zhu J, Wang KZ and Chu CT: After the banquet: mitochondrial biogenesis, mitophagy and cell survival. Autophagy 9: 1663-1676, 2013.

49. Piantadosi CA and Suliman HB: Redox regulation of mitochondrial biogenesis. Free Radic Biol Med 53: 2043-2053, 2012.

50. Wredenberg A, Wibom R, Wilhelmsson $\mathrm{H}$ et al: Increased mitochondrial mass in mitochondrial myopathy mice. Proc Natl Acad Sci USA 99: 15066-15071, 2002.

51. Puigserver $\mathrm{P}$ and Spiegelman BM: Peroxisome proliferatoractivated receptor-gamma coactivator 1 alpha (PGC-1 alpha): transcriptional coactivator and metabolic regulator. Endocr Rev 24: 78-90, 2003. 
52. Lin J, Handschin C and Spiegelman BM: Metabolic control through the PGC-1 family of transcription coactivators. Cell Metab 1: 361-370, 2005.

53. Scarpulla RC: Transcriptional paradigms in mammalian mitochondrial biogenesis and function. Physiol Rev 88: 611-638, 2008.

54. Malik AN and Czajka A: Is mitochondrial DNA content a potential biomarker of mitochondrial dysfunction? Mitochondrion 13: 481-492, 2013

55. Cormio A, Guerra F, Cormio G, Pesce V, et al: The PGC-1alpha-dependent pathway of mitochondrial biogenesis is upregulated in type I endometrial cancer. Biochem Biophys Res Commun 390: 1182-1185, 2009.

56. Montgomery BE, Daum GS and Dunton CJ: Endometrial hyperplasia: a review. Obstet Gynecol Surv 59: 368-378, 2004.

57. Cormio A, Guerra F, Cormio G, et al: Mitochondrial DNA content and mass increase in progression from normal to hyperplastic to cancer endometrium. BMC Res Notes 5: 279, 2012.

58. Mayr JA, Meierhofer D, Zimmermann F, et al: Loss of complex I due to mitochondrial DNA mutations in renal oncocytoma. Clin Cancer Res 14: 2270-2275, 2008.

59. Gasparre G, Kurelac I, Capristo M, et al: A mutation threshold distinguishes the antitumorigenic effects of the mitochondrial gene MTND1, an oncojanus function. Cancer Res 71: 6220-6229, 2011.

60. Kurelac I, MacKay A, Lambros MB, et al: Somatic complex I disruptive mitochondrial DNA mutations are modifiers of tumorigenesis that correlate with low genomic instability in pituitary adenomas. Hum Mol Genet 22: 226-238, 2013.

61. Gasparre G, Bonora E, Tallini G and Romeo G: Molecular features of thyroid oncocytic tumors. Mol Cell Endocrinol 321: 67-76, 2010.

62. Gasparre G, Romeo G, Rugolo M and Porcelli AM: Learning from oncocytic tumors: Why choose inefficient mitochondria? Biochim Biophys Acta 1807: 633-642, 2011.

63. Gasparre G, Porcelli AM, Lenaz G and Romeo G: Relevance of mitochondrial genetics and metabolism in cancer development. Cold Spring Harb Perspect Biol 5, pii: a011411, 2013.

64. Hirst J, King MS and Pryde KR: The production of reactive oxygen species by complex I. Biochem Soc Trans 36: 976-980, 2008 .
65. Murphy MP: How mitochondria produce reactive oxygen species. Biochem J 417: 1-13, 2009.

66. Papa S, De Rasmo D, Technikova-Dobrova Z, et al: Respiratory chain complex I, a main regulatory target of The cAMP/PKA pathway is defective in different human diseases. FEBS Lett 586: 568-577, 2012.

67. Porcelli AM, Ghelli A, Ceccarelli C, et al: The genetic and metabolic signature of oncocytic transformation implicates HIF1alpha destabilization. Hum Mol Genet 19: 1019-1032, 2010.

68. Calabrese C, Iommarini L, Kurelac I, et al:Respiratory complex I is essential to induce a Warburg profile in mitochondria-defective tumor cells. Cancer Metab 1: 11, 2013.

69. Chen JQ, Cammarata PR, Baines CP and Yager JD: Regulation of mitochondrial respiratory chain biogenesis by estrogens/ estrogen receptors and physiological, pathological and pharmacological implications. Biochim Biophys Acta 1793: 1540-1570, 2009.

70. Chen JQ and Russo J: Mitochondrial oestrogen receptors and their potential implications in oestrogen carcinogenesis in human breast cancer. J Nutr Environ Med 17: 76-89, 2008.

71. Mattingly KA, Ivanova MM, Riggs KA, Wickramasinghe NS, Barch MJ and Klinge CM: Estradiol stimulates transcription of nuclear respiratory factor-1 and increases mitochondrial biogenesis. Mol Endocrinol 22: 609-622, 2008.

72. Felty Q, Xiong WC, Sun D, Sarkar S, Singh KP, Parkash J and Roy D: Estrogen induced mitochondrial reactive oxygen species as signal-transducing messengers. Biochemistry 44: 6900-6909, 2005.

73. Stankov K, Biondi A, D'Aurelio M, Gasparre G, Falasca A, Romeo G and Lenaz G: Mitochondrial activities of a cell line derived from thyroid Hürthle cell tumors. Thyroid 16: 325-331, 2006.

74. Guerra F, Kurelac I, Magini P, Cormio A, Santini D, Ceccarelli C and Gasparre G: Mitochondrial DNA genotyping reveals synchronous nature of simultaneously detected endometrial and ovarian cancers. Gynecol Oncol 122: 457-458, 2011.

75. Guerra F, Girolimetti G, Perrone AM, et al: Mitochondrial DNA genotyping efficiently reveals clonality of synchronous endometrial and ovarian cancers. Mod Pathol 39: 1412-1420, 2014. 